\title{
PATOLOGÍAS FORESTALES Y CAMBIO GLOBAL: GLOBALIZACIÓN, CAMBIO CLIMÁTICO Y CUESTIONES LEGALES
}

\author{
Julio Javier Diez Casero \\ Universidad de Valladolid. Instituto de Investigación en Gestión Forestal Sostenible. Escuela Técnica \\ Superior de Ingenierías Agrarias. Avenida de Madrid 44. 34071-PALENCIA (España). Correo electrónico: \\ jdcasero@pvs.uva.es
}

\begin{abstract}
Resumen
El denominado Cambio Global está afectando al modo de vida de la población, a su economía, al mercado laboral, los mercados agrícolas y, como no, también al sector forestal. En lo que concierne al tema de las patologías forestales, en las últimas décadas se ha producido un considerable incremento en la tasa de aparición de nuevas enfermedades (primeras citas, "first reports") a escala mundial, debido entre otras razones al rápido incremento del comercio de madera y de material forestal de reproducción por efecto de la globalización. Enfermedades como el chancro resinoso del pino causado por Fusarium circinatum, o el nematodo de la madera del pino, Bursaphelenchus xylophilus, han aparecido recientemente en nuestro país por esta vía. Por otra parte, en los últimos años están apareciendo nuevas enfermedades de origen complejo (denominadas enfermedades de decaimiento, o "declines") que se relacionan con la acción simultánea de diversos agentes bióticos, pero donde los factores abióticos relacionados con el clima toman una importancia preponderante. La seca de la encina (en la que la influencia de Phytophthora spp. no se discute), o el decaimiento del pino resinero (Pinus pinaster) podrían ser ejemplos de este tipo. Todos estos problemas se ven amplificados, en los casos más graves, por una administración forestal en temas sanitarios no suficientemente apoyada, mal financiada, demasiado disgregada tanto en el ámbito global, como nacional o regional, que se ve limitada en ocasiones para abordar el manejo del creciente número de patologías forestales que están apareciendo.
\end{abstract}

Palabras clave:

\section{INTRODUCCIÓN}

El número de patologías forestales está aumentando de forma exponencial durante las últimas décadas al tiempo que su impacto parece ser cada vez mayor. Así, a enfermedades ya tradicionales como la tinta y el chancro del castaño, o la grafiosis de los olmos han seguido otras muchas enfermedades como la seca de la encina, el decaimiento del aliso, el chancro resinoso del pino, el decaimiento del pino pinaster, la gremmeniela, el nematodo de la madera del pino...así como otras muchas que pudieran aparecer en un futuro en nuestro país como el decaimiento del fresno (Hymenosciphus pseudoalbidus) o las enfermedades causadas por Phytophthora pinifolia o Leptographium wagenerii. Pudiera pensarse que el incremento en la actividad investigadora llevado a cabo en este campo durante las últimas décadas bien pudiera ser una de las explicaciones a este incremento pero, tres son los factores directamente implicados en ello: (1) la globalización, (2) 
el cambio climático y (3) la falta de una legislación efectiva a escala global, que evite la introducción de los organismos invasivos entre países.

\section{INFLUENCIA DE LA GLOBALIZACIÓN EN LA SANIDAD FORESTAL}

El cambio global que en la actualidad estamos experimentado está afectando al modo de vida de la población, que tiene que adaptarse a los nuevos retos que esto supone; entre ellos el enorme cambio económico que estamos sufriendo, que por el momento se traduce en una crisis sin precedente a escala mundial. Este cambio está modificando el mercado laboral en algunos países, que están reduciendo de una forma drástica el número de puestos de trabajo a escala global y condenando a un porcentaje importante de la población a la lacra del desempleo, lo que se traduce en un incremento de la presión social. Los mercados agrícolas no son inmunes a todo esto, y en la actualidad están sufriendo fluctuaciones que hacen difícil hacer previsiones macro-económicas a escala global, al estar de forma continua a merced de los especuladores. En esta misma línea el sector forestal también está sufriendo todos estos avatares, en mayor medida incluso que la agricultura, al poseer un producto de mercado con un valor muy limitado, e incluir dentro de sus valores productos intangibles, a veces difíciles de evaluar.

En lo que concierne al tema de las patologías forestales, la globalización, y el incremento extraordinario del comercio de madera y material forestal para reproducción asociado, ha provocado un aumento considerablemente en la tasa de aparición de nuevas enfermedades (Figura 1). Esto puede visualizarse fácilmente revisando el elevado número de primeras citas ("first reports") a escala mundial en los últimos años. Como hecho anecdótico, algunas de las revistas se han visto tan desbordadas por este tipo de artículos, que han decidido abandonar su publicación, o crear nuevas revistas centradas únicamente en este tipo de informaciones (e.j. Australasian Plant Disease Notes). La importancia de este tipo de publicaciones es considerable ya que, como en medicina, si un especialista no es capaz de diagnosticar la enfermedad que un determinado paciente tiene, será muy difícil que pueda curarle.
Enfermedades como el chancro resinoso del pino causado por Fusarium circinatum, o el nematodo de la madera del pino, Bursaphelenchus xylophilus son un buen ejemplo de organismos invasivos recientemente introducidos en nuestro país a través de semillas y madera, respectivamente. Una vez establecida, la enfermedad causada por Fusarium circinatum afecta tanto a material vegetal para reproducción (semillas y plantas de vivero) como a árboles adultos en el monte. Pero es sin lugar a dudas, el material de reproducción la mejor vía de dispersión de este hongo. Así, la enfermedad se supone que fue introducida a través de la importación de semilla de pino radiata (posiblemente de forma legal) desde California. La península de Monterrey, en este estado americano, es la zona natural de distribución de esta especie forestal, la más plantada en el mundo. Por ello, la importación de semilla de este área suele ser frecuente, en busca de la variabilidad necesaria para la mejora de las plantaciones. Sin embargo, este tipo de importaciones entrañan un enorme peligro, máxime cuando la zona lleva ya varias décadas afectada por el chancro resinoso. Además, la detección del patógeno en las semillas es bastante complicada, al existir niveles de infección extremadamente bajos (a veces sólo 1 semilla por cada 500). En estas condiciones es muy difícil asegurar la asepsia del material vegetal a introducir, lo que representa un peligro para las plantaciones a escala nacional.

La enfermedad del nematodo de la madera del pino, causada por Bursaphelenchus xylophilus, fue introducida en Europa a través de Portugal a finales de los años noventa. La importación de madera colonizada por el nemátodo, y su posterior inoculación en el monte por el cerambícido vector Monochamus galloprovincialis, parece ser el origen de esta introducción. Tras un periodo de cuarentena, donde se suponía recluida únicamente a la península de Setúbal, en las proximidades de Lisboa, la enfermedad escapó al control detectándose posteriormente en decenas de puntos a lo largo de toda la geografía lusa. La especie afectada durante este periodo ha sido el pino resinero, siendo el pino piñonero, presente en muchos de los focos, resistente a la enfermedad. En los últimos años, se han detectado positivos en dos árboles en 

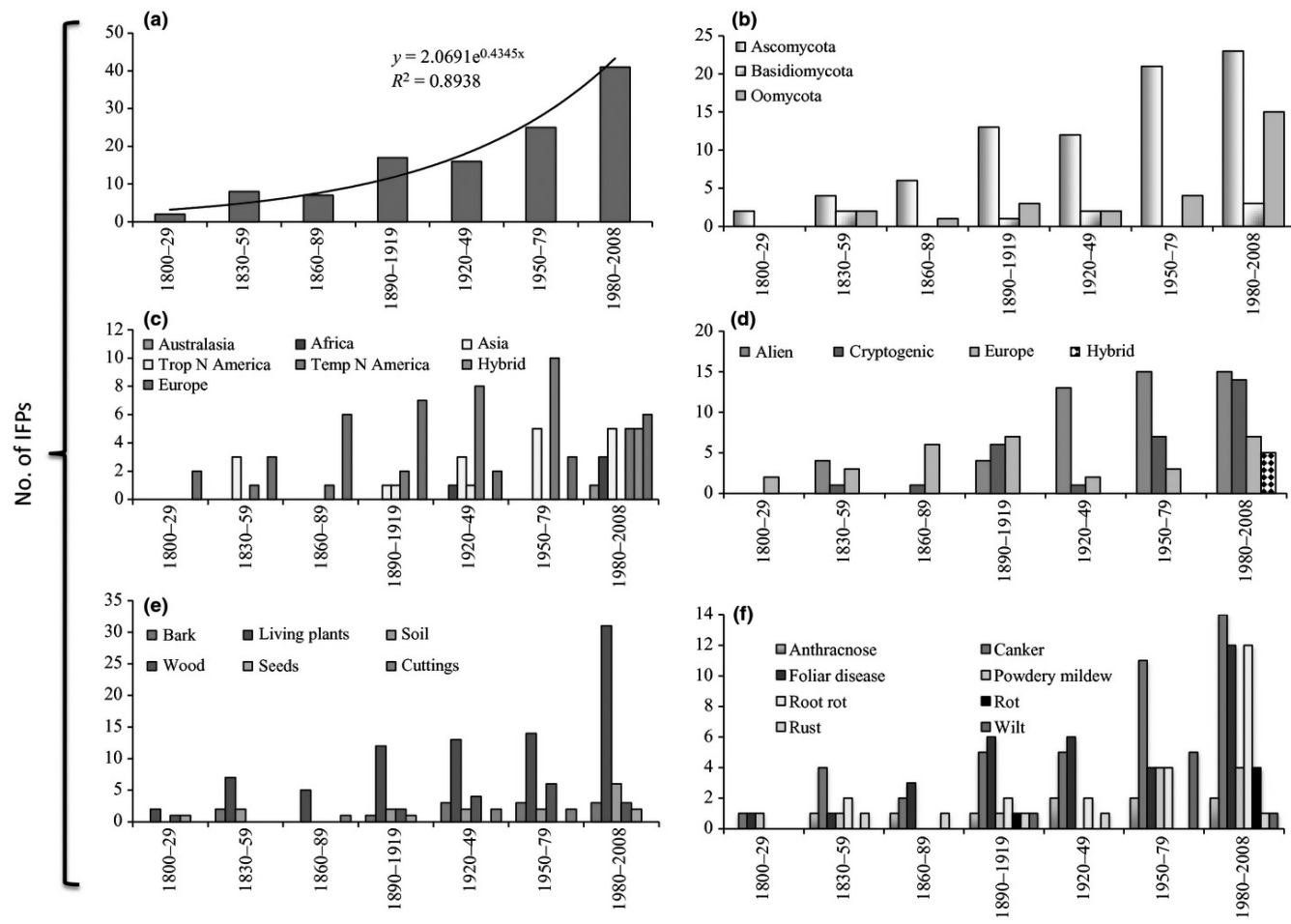

Figura 1. Patógenos introducidos en Europa desde 1800 a 2008 (SANTINI et al., 2013)

Extremadura y uno en Galicia (Real Decreto 58/2005, de 21 de enero), aunque la enfermedad parece ser que no está aún instalada en el monte.

La globalización está homogeneizando parte de los procesos y reglas económicas, en busca de una economía de libre mercado. Pero esta homogeneización no es general ni total en muchos aspectos, lo que esta originando la aparición de nuevos problemas. Así, al igual que la creación de una Unión Europea con una moneda común pero con gobiernos nacionales con prácticamente las mismas competencias, y la falta de un mercado de deuda común o una unión bancaria real está provocando tensiones que están afectando a países con dificultades económicas (caso de Grecia, Italia o España) la falta de leyes fitosanitarias comunes en este mercado global, o al menos efectivas a esta escala está ocasionando problemas de coordinación en el ámbito de la Sanidad Forestal. Esto permite en ocasiones, que los intereses económicos nacionales tengan más peso que los intereses globales de los estados, produciéndose a veces una falta de sinceridad entre países a la hora de declarar sus problemas fitosanitarios, que asumen sin coste alguno, sin ninguna responsabilidad, su ocultación en aras de evitar un perjuicio económico a sus intereses. Este comportamiento anti-solidario está provocando que las enfermedades se distribuyan sin freno alguno de país a país, sin visos en el horizonte de poder reducir esta lacra. Este efecto puede producirse a nivel internacional, o incluso a veces intranacional, y está mucho más acentuado en países con un estado federal o autonómico donde la responsabilidad fitosanitaria forestal se encuentra transferida, como es el caso de España.

El material forestal de reproducción (Figura 2) representa la vía de dispersión más común para muchas enfermedades. Así, las semillas son el medio de dispersión más efectivo a larga distancia mientras que las plantas de viveros son la vía de dispersión idónea a media y corta distancia. Existe 


\section{Cambio Global Globalización}

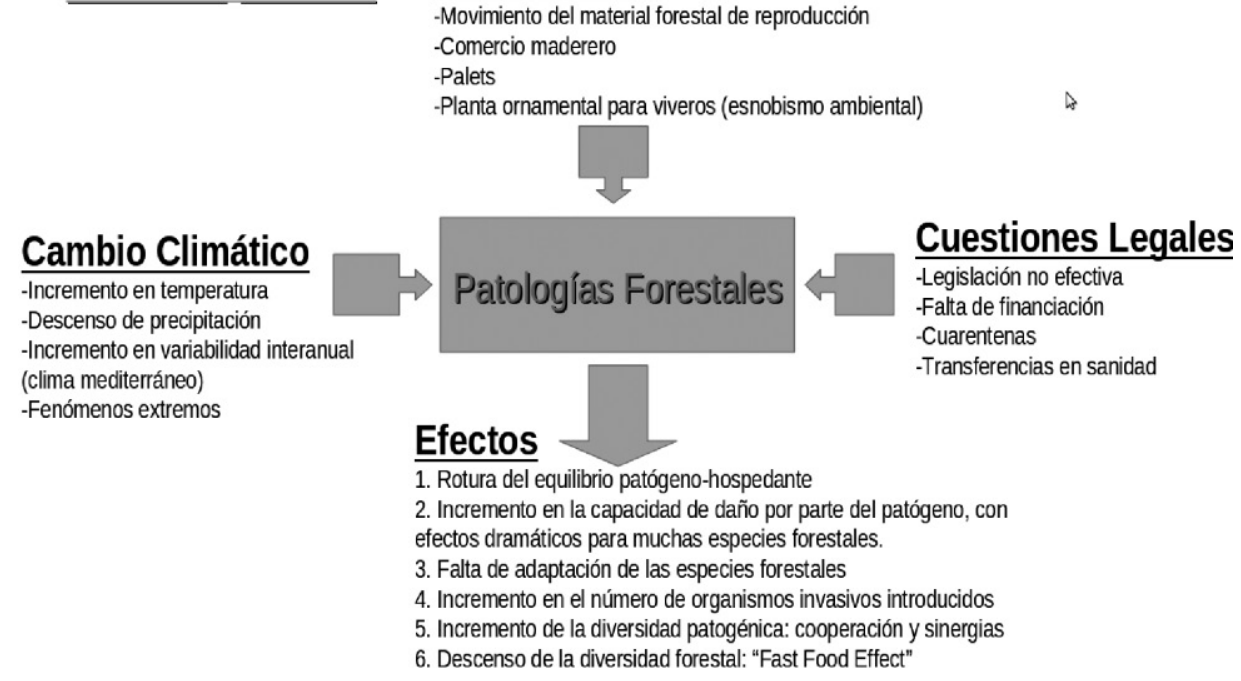

Figura 2. Representación esquemática de los efectos causados por el cambio global sobre las patologías forestales

un estudio realizado en viveros forestales de toda Europa (THOMAS JUNG, comunicación personal) que ha analizado las especies de Phytophthora patógenas existentes, y ha encontrado que más de un $80 \%$ de los viveros están vendiendo planta infectada con estas especies. Este tipo de resultados hacen plantearse el actual sistema productivo de planta para reforestación por las altas posibilidades de transferir las enfermedades, recopiladas en los viveros, directamente al campo. Esto puede sonar extraño, ya que es un sistema de producción universal ampliamente probado, utilizado y efectivo, pero en la actualidad ya existen diversos grupos que se encuentran ensayando diversos sistemas de siembra de semillas de forma directa en monte, así como dispositivos para proteger la semilla instalada en el monte en estas condiciones (REQUE-KILCHENMANN, 2013). Quizás sea ya muy difícil cambiar este sistema productivo, aunque tampoco es tan lejana la época en la que el patrimonio forestal del estado situaba los viveros sobre eras en el monte (sin envase) para producir la planta que iba a usarse en la repoblación de ese mismo monte. La existencia de viveros con producción de planta en envase para repoblación de zonas distantes es relativamente reciente. El alto número de patologías que se están transfiriendo desde el vivero al monte requiere de un cambio radical en el sistema productivo que habrá que discutir durante la presente década.

El importante papel de los viveros forestales en la dispersión de enfermedades se ha puesto de manifiesto con la enfermedad causada por Fusarium circinatum. La introducción en nuestro país a través de semilla procedente de California, el desconocimiento de la enfermedad por parte de los productores por la falta de una declaración oficial, y la distribución de la planta proveniente de viveros infectados, antes de la declaración de la enfermedad, explica la rápida dispersión a nivel regional. Es por ello que el conocimiento de la existencia de una enfermedad forestal y su pronta declaración por parte de las autoridades fitosanitarias es vital para evitar la dispersión a amplias zonas.

Pese a la importancia de los viveros en la dispersión de enfermedades y la necesidad inminente de regulación, este tipo de acciones no suelen contar con el apoyo de aquellos países en los cuales este sector representa un aporte económico esencial para su economía. Sin embargo, hay que pensar de una forma realista lo que realmente representa para la economía las producciones de cuatro a cinco viveros en relación con lo que representa la de los bosques de todo un país o un continente. 
Es necesario actuar para evitar la gran pandemia que representa la introducción reiterada de nuevas enfermedades año tras año. Pese, a la falta de voluntad real de algunos organismos $u$ organizaciones es necesario de un mayor control del trasporte de material vegetal, y sobre todo del material vegetal de reproducción, causante de gran parte de las nuevas introducciones de patógenos. Llevándolo al extremo, de la misma forma, que el comercio de mapaches no representa un porcentaje demasiado importante del PIB de los países evolucionados, y del perfectamente podría prescindirse al no aportar demasiado a la economía nacional, pudiendo por otra parte provocar problemas de salud pública y ecológicos (como el que ya existe en algunas comunidades como Madrid), existen diferentes mercados residuales de plantas exóticas de los que perfectamente podríamos prescindir para evitar problemas de nuevas introducciones de patógenos. Estas plantas exóticas suelen venir acompañadas de patógenos exóticos, que una vez establecidos es imposible eliminar. Es por ello, que debiéramos de plantearnos una suspensión temporal de estos mercados de planta ornamental en aras de una mayor protección de nuestros bosques. Este es el principal objetivo de la "Declaración de Montesclaros", redactada en el año 2011 dentro de la reunión del IUFRO "Global changes and forest diseases: new treaths new strategies" en este monasterio cántabro, y que trata de concienciar a la población y estamentos con competencias en sanidad forestal sobre la necesidad de esta regulación para mitigar la entrada constante de especies invasoras que está teniendo lugar a escala global.

\section{EL CAMBIO CLIMÁTICO Y SU INFLUENCIA SOBRE LAS PATOLOGÍAS FORESTALES}

La alteración de las condiciones climáticas durante las últimas décadas parece una realidad indiscutible. El cambio climático afecta a diversos factores como la precipitación, el ciclo de carbono, la acidificación del océano o el nivel del mar entre otros (MeEHL et al., 2007). Es sin embargo la temperatura el indicador mas utilizado para medir las tendencias del cambio climá- tico, también denominado calentamiento global. Se espera que la temperatura de la tierra sufra un incremento en temperatura de $2-4,5^{\circ} \mathrm{C}$ durante este siglo. Los cambios en los valores de precipitación parecen ser variables en función del tipo de clima: descenso y mayor variabilidad en ambientes mediterráneos, y aumento en las zonas más atlánticas (GIORGI, 2006; GIORGI \& LIONELLO, 2008). El cambio climático es un problema íntimamente ligado al desarrollo, asociado a nuestro modelo de crecimiento basado en la quema de combustibles fósiles y patrones de consumo y producción poco eficientes. Constituye un reto sin precedentes dar una respuesta eficaz a las causas que originan el problema por la dificultad que supone. Para contribuir a su mitigación es necesario reducir un $50-85 \%$ las emisiones globales de $\mathrm{CO}_{2}$ en el año 2050 respecto a las del año 2000 para evitar males mayores. Aunque esta reducción parece difícil, máxime en el escenario actual de crisis económica que estamos sufriendo.

En los últimos años están apareciendo nuevas enfermedades de origen complejo (denominadas enfermedades de decaimiento, o "declines") que parecen estar relacionadas con el cambio climático. En estas patologías aparecen asociados diversos agentes bióticos (hongos, oomicetos...), pero también factores abióticos de tipo climático (sequías, incremento de temperaturas...). Algunos de estos agentes predisponen al árbol a sufrir la enfermedad, otros aceleran sus síntomas, y por último otros que causan la muerte del árbol (MANION, 1991). La asociación de todos estos agentes con la enfermedad complica mucho el diagnóstico, ya que es bastante difícil establecer la importancia real de cada uno de ellos en el daño global del árbol. Esto hace que el origen, asociación y epidemiología de muchas de estas enfermedades siga aún sin esclarecerse, a veces décadas después de las primeras citas. La seca de la encina (en la que la influencia de Phytophthora spp. no se discute), o el decaimiento del pino resinero son ejemplos de enfermedades de este tipo, donde el efecto de clima, y sus variaciones podrían estar implicados en la enfermedad.

La seca de la encina es una patología que apareció a principios de los años 80 en el sur de la península ibérica afectando a esta especie 
(Quercus ilex) y también al alcornoque ( $Q$. suber). Suele afectar a rodales que a veces superan cientos de hectáreas, donde provoca dos tipos de síntomas: (1) muerte súbita, al necrosar completamente la copa en un periodo de unos pocos meses (las hojas se vuelven marrones y se mantienen pegadas a la copa como si fueran marcescentes), y (2) muerte progresiva, donde la enfermedad provoca la muerte de los árboles a lo largo de varios años (síntomas de puntisecado, con gran cantidad de ramas secas en la copa). Como el propio nombre de la enfermedad indica, apareció asociada a largos periodos de sequía que se produjeron en la península a principios de los años 80. Estos largos periodos de sequía seguidos de periodos de inundación son en la actualidad cada vez más frecuentes y parecen ser el resultado de fluctuaciones en el clima acaecidos a lo largo del último siglo. El oomiceto Phytophthora cinnamomi (y otros como $P$. gonapodyides) son los agentes bióticos más importantes asociados a esta enfermedad.

El decaimiento del pino pinaster (Figura 3) es una enfermedad que se viene produciendo durante las dos últimas décadas y que se caracteriza por una inusual transparencia de copa, reducción del tamaño de las acículas, decoloración del follaje, y la muerte temprana del árbol. En los sitios fuertemente afectados la mortalidad puede afectar a más del $60 \%$ de los árboles de la parcela. Esta enfermedad aparece asociada a presencia de muérdago, y hongos como Ophiostoma minus y Heterobasidion annosum. Debido a la rapidez, y a veces homogeneidad de aparición de los síntomas, esta enfermedad también se ha asociado a episodios de sequía en suelos arenosos.

El cambio climático también parece estar afectando al desarrollo de los insectos plaga, así como a la gravedad de los daños por estos provocados. De esta forma, brotes eruptivos como los producidos por Dendroctonus ponderosae en Canadá también parecen estar relacionados con los efectos de este cambio. Este escolítido perforador es el causante de la mortalidad de millones de hectáreas de coníferas en Canadá (PAGE et al., 2013), siendo en este momento la plaga forestal más importante en este país. Según los últimos estudios llevados a cabo, parece ser que un pequeño incremento en la temperatura media de la zona, evita que las larvas que se encuentran en la corteza de los árboles se congelen, incrementando así fuertemente su población. Este hecho permite sobrepasar el umbral endémico población que de esta forma se convierte en epidémico, provocando brotes eruptivos que son capaces de atacar a los árboles sanos.

La modificación de las condiciones ambientales ejerce una gran influencia sobre las enfer-

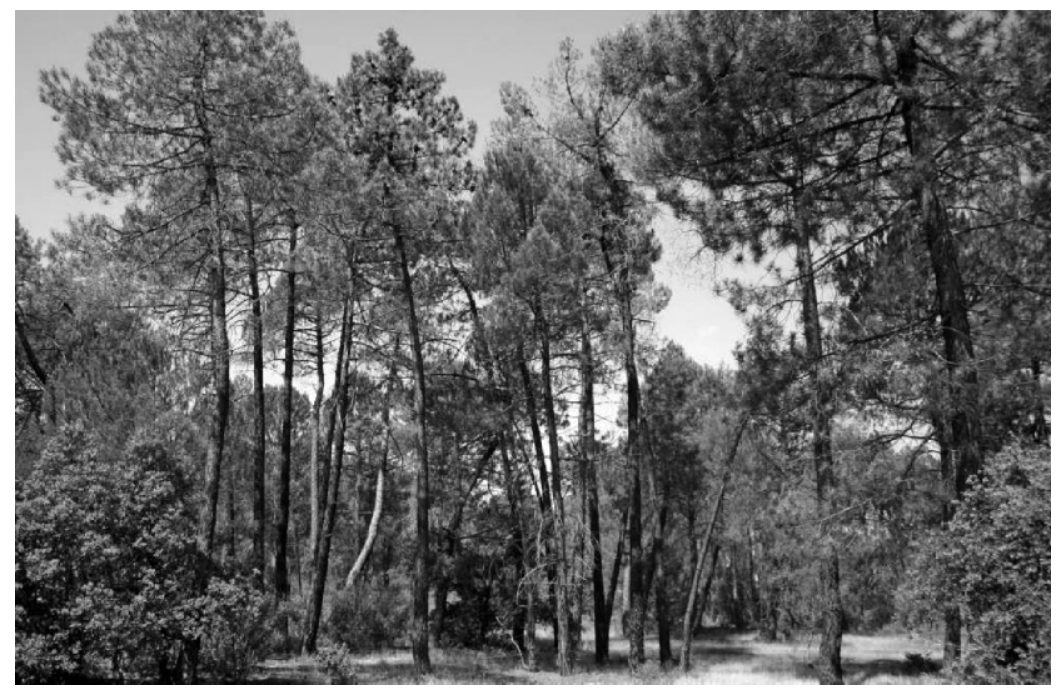

Figura 3. Ejemplares de Pinus pinaster afectados por decaimiento 
medades forestales. Diversos estudios han puesto de manifiesto que el cambio climático va a afectar sin lugar a dudas al impacto de las enfermedades vegetales, teniendo en cuenta que la frecuencia de episodios climáticos extremos como sequías, inundaciones, fluctuaciones en las temperaturas máximas, y la frecuencia de tormentas se van a incrementar durante las próximas décadas (HARVELl et al., 2002; LA PORTA et $a l .$, 2008; STURROCK et al., 2011). Esto altera el comportamiento de los agentes patógenos, al tiempo que puede debilitar las capacidades defensivas frente a enfermedades de ciertas especies hospedantes adaptadas al medio durante años. La capacidad de adaptación del patógeno suele ser mucho más rápida que la de los hospedantes que generalmente poseen generaciones longevas, por lo que esté último será el más perjudicado con este cambio. Sin embargo, el efecto del cambio climático sobre los diversos patógenos (positivo, negativo o neutral) dependerá de las características de cada patógeno y su hospedante (CHAKRABORTY et al., 2000; BOLAND et al., 2004; YÁÑEZ-LóPEZ et $a l .$, 2012).

Es necesario desarrollar más trabajos de investigación para conocer la intensidad y dirección de este cambio, así como adelantarnos a los diversos escenarios que aparecerán en un futuro. Proyectos transnacionales como el REINFFORCE (Interreg IV B) tratan de dar respuestas a todo esto a través de la instalación de arboretum y zonas de demostración para estudiar el efecto del cambio climático sobre los bosques.

\section{CUESTIONES LEGALES, SANIDAD FORESTAL Y CAMBIO GLOBAL}

Los cambios en las leyes del comercio global, y en la administración fitosanitaria también están afectando al comercio de productos forestales. Así, decisiones como la supresión de fronteras acaecida tras la consecución del mercado único europeo ha sido nefasta en el ámbito de la sanidad forestal. Pese a que los controles fitosanitarios entre países tienen aún vigencia y en cierta medida se han aumentado, la facilidad para transportar material vegetal fuera de control es en la actualidad mucho mayor, incrementándose de esta forma las posibilidades de introducción fortuita de nuevas patologías. Por otra parte, los daños ocasionados por la introducción de especies invasivas se ven amplificados a veces, por una administración forestal en temas sanitarios no suficientemente apoyada, donde su importancia relativa en relación, por ejemplo con la medicina, es mucho menor: no se le concede la importancia que realmente tiene, pese a los graves problemas ecológicos y económicos que comporta. De esta forma, la financiación que se dedica a la gestión administrativa de las patologías forestales es claramente insuficiente pese a la importancia que la salud de nuestros bosques posee.

Por otra parte, los diversos organismos nacionales e internacionales responsables de la gestión de la sanidad forestal presentan a veces niveles de disgregación elevados, lo que perjudica la sincronización de las respuestas ante problemas fitosanitarios. Así a la hora de realizar inspecciones, erradicaciones o contenciones para luchar contra una determinada patología, la colaboración, sincronización y ayuda entre administraciones es vital para el éxito de la campaña. Sin embargo, muchas veces esta coordinación entre países, e incluso a veces en un sistema descentralizado como el nuestro, entre administraciones autonómicas, deja mucho que desear, redundando en un perjuicio para el sistema y para el interés general. La cesión de ciertas competencias en sanidad forestal por parte de los estados a organismos supranacionales, como la UE está siendo positivo para el control de las enfermedades. De esta forma, los intereses nacionales se están relegando a un segundo plano, tapados por los generales, con una visión más amplia e integradora. Es por ello que resulta vital que todos los organismos relacionados con la sanidad forestal no tengan problemas en ceder competencias a organismos de mayor entidad, que tomen las decisiones de forma aséptica y coordinada con la vista puesta en el interés general, o como mínimo que se organicen adecuadamente y compartan información. Sin embargo, desafortunadamente muchos de estos organismos $\mathrm{u}$ organizaciones siguen teniendo una importancia muy limitada, a veces sin capacidad de ejecución, y con enormes limitaciones presupuestarias, por lo que poco pueden hacer para evitar la libre dispersión de enfermedades más que actuar como centros de diagnóstico. 
De otra parte, también hay que destacar que en ocasiones las posibilidades de reacción frente a la introducción de ciertas enfermedades son difíciles de ejecutar. Así, como es difícil de evitar que un pescador sin escrúpulos introduzca de por libre una nueva especie acuática en un cauce, provocando con el paso del tiempo un desastre ecológico sin precedentes en la ictiofauna, es prácticamente imposible controlar acciones individuales, que a veces son simplemente causa de irresponsabilidad o desconocimiento, y que pueden ocasionar la introducción de una nueva enfermedad en el monte. Es por ello, que la concienciación de la población frente a este tipo de problemas fitosanitarios es muy importante, aunque aún una tarea pendiente.

Es necesario incrementar los esfuerzos para intentar mitigar los efectos del cambio global sobre la sanidad de las masas forestales, y la aparición de nuevas enfermedades. Así, los gobiernos europeos, nacionales y autonómicos deben de procurar una mayor financiación para la disciplina de la sanidad forestal. Por otra parte, las administraciones europeas, nacionales y autonómicas responsables de la sanidad forestal deben de procurar una mayor sincronización entre ellas, estableciendo mecanismos fluidos para el intercambio de información. A su vez, los legisladores deben de proponer reglas que sean factibles y realmente útiles y aplicables desde la realidad actual de forestales y propietarios. Y por último, los técnicos y científicos deben de trabajar en éstas líneas mediante investigación aplicada tratando de mejorar métodos de diagnóstico, seguimiento y control de enfermedades, así como participar en los comités consultivos, proponiendo procedimientos de inspección, erradicación y contención efectivos, y realmente aplicables. Es también, obligación de este gremio, el presionar para que los gobiernos y entes locales dediquen más esfuerzos a la contención de estas nuevas enfermedades. En esta línea se ha trabajado en la declaración de Montesclaros.que fue redactada por un grupo de más de 70 científicos expertos en patología forestal representando a 17 países que asistieron a la reunión internacional del IUFRO (WP 7.02.02) que tuvo lugar en el Monasterio de Montesclaros, Cantabria (España) del 23 al 27 de mayo de 2011. En la actualidad la declaración se encuentra ratificada y apoyada por 142 investiga- dores pertenecientes a 31 países. El texto en castellano (el documento original en inglés fue traducido a 13 idiomas) aparece a continuación.

\section{LA DECLARACIÓN DE MONTESCLAROS}

Escrita por un grupo de más de 70 científicos expertos en patología forestal (representando a 17 países) que asistieron a la reunión internacional del IUFRO ${ }^{\prime}$-que tuvo lugar en el Monasterio de Montesclaros, Cantabria (España) del 23 al 27 de mayo de 2011.

Como científicos con una amplia experiencia en el estudio de enfermedades forestales, queremos poner de manifiesto que el transporte indiscriminado de material vegetal está incrementando en gran medida el número de patologías forestales a escala global. Esta evidencia está basada en la gran cantidad de de especies patógenas exóticas y plagas recientemente introducidas en bosques y plantaciones forestales en todas las partes del planeta, hecho sin precedentes en la historia de la humanidad. Por ello, proponemos que todo comercio de plantas y productos vegetales con riesgo para el ecosistema forestal y sin importante beneficio económico $^{2}$ se interrumpa de inmediato.

${ }^{I}$ IUFRO = Unión Internacional de Organizaciones de Investigación Forestal (www.iufro.org)

${ }^{2}$ Se considera todo comercio internacional de planta ornamental en envase forestal, así como árboles adultos para restauración forestal con bajo beneficio en términos económicos pero alto riesgo para la salud forestal. Hay que tener en cuenta que la producción de planta a bajo precio en diversos países para su plantación en otros muy diferentes y alejados proporciona unos beneficios netos muy marginales para el área, pero es la perfecta vía de dispersión para enfermedades y plagas. Además, el comercio internacional de otro material vegetal (madera para embalajes, astillas, trozos de corteza...) debe de ser analizado con detalle y estrictamente regulado.

\section{CONCLUSIÓN FINAL}

La aparición cada vez más frecuente de plagas y enfermedades forestales es un "goteo" continuo que por el momento no tiene visos de parar. La entrada de organismos invasivos ha experi- 
mentado un crecimiento exponencial, ligado entre otras cosas, al enorme incremento de comercio de materiales vegetales. Teniendo en cuenta que una vez introducida una especie vegetal es prácticamente imposible su erradicación, es necesario un incremento en las inspecciones de todas las importaciones de materiales vegetales.

En relación con el incremento de las patologías asociadas al cambio climático, poco se puede hacer, salvo la reducción de las emisiones de $\mathrm{CO}_{2}$ a la atmósfera con el fin de tratar de mitigar los problemas del efecto invernadero. Por otra parte, es necesario potenciar los estudios relativos a las nueva situación derivada de este cambio, así como el nivel adaptación que tendrán las especies en las nuevas condiciones venideras. Iniciativas europeas como el proyecto REINFFORCE, que ha establecido una red de 38 arboretum y 41 zonas de demostración a lo largo de toda la Europa atlántica para el estudio de la adaptación de las especies forestales al cambio climático, aportarán luz sobre este tema en los próximos años.

Más acciones para mitigar la dispersión de enfermedades se pueden llevar a cabo en el tema de legislación. Pese a la influencia que una declaración de cuarentena puede tener sobre las exportaciones de madera y productos vegetales, y su incidencia en la economía del país, en ningún caso las administraciones comunitarias, nacionales o regionales pueden permanecer impasibles ante la introducción de una nueva patología. Los patógenos no entienden de economía, política o de fronteras, y la falta de acción por cualquiera de estos motivos es el escenario perfecto para una rápida dispersión de una enfermedad, una vez introducida en un país. Desde la primera noticia de su existencia en otros países cercanos habría que organizar a todos los sectores implicados para tratar de impedir su aparición, mediante el establecimiento de protocolos de inspección y reglas efectivas y realistas (que sean factibles de ser cumplidas). Esta es en general la fase que suele estar más descuidada, por la cantidad de enfermedades existentes, siendo a su vez la más importante ya que una vez que una enfermedad es introducida en un país, se quedará para siempre. La falta de coordinación entre administraciones es el primer escollo que hay que salvar, aunque suele ser a veces el más difícil, por los típicos pro- blemas de competencias entre estamentos. Sin embargo, hay que tener en cuenta que la salud del arbolado es lo que tiene siempre que primar en cualquier decisión, ya que el restablecimiento de las condiciones originarias de un bosque tras un proceso de enfermedad suele durar siglos, y en muchas ocasiones nunca llega a recuperarse.

La experiencia nos dice que lo más normal es que con el tiempo todo tienda al equilibrio. Por ello, la introducción de nuevos agentes patógenos podría contribuir con el paso del tiempo a la consecución de un ecosistema más estable (teoría del vigor híbrido). Pero aún así, tenemos que ser conscientes que esta llegada de nuevos organismos patógenos va a ocasionar profundos cambios en el ecosistema forestal, provocando la desaparición de gran cantidad de plantaciones y bosques naturales, lo que sin duda acarreará graves pérdidas económicas y ecológicas a corto plazo como ya estamos viendo. Este cambio es imparable, pero bien es cierto que está en nuestra mano la posibilidad de su mitigación, aumentando de esta forma las posibilidades de reacción de las especies más susceptibles. El cambio global no tiene marcha atrás, y este cambio también implica una alteración general en los ecosistemas forestales, donde la homogeneidad, ganara espacio en detrimento de la diversidad (teoría del Fast Food Effect).

\section{BIBLIOGRAFÍA}

Boland, G.J.; Melzer, M.S.; Hopkin, A.; Higgins, V. \& NAssuth, A.; 2004. Climate change and plant diseases in Ontario. Can. J. Plant Pathol. 26: 335-350.

Chakraborty, S.; Tiedemann, Av. \& Teng, P.S.; 2000. Climate change: potential impact on plant diseases. Environ. Pollut. 108: 317-326.

GioRgI, F.; 2006. Climate change hot-spots. Geophys. Res. Lett. 33(8): L08707.

Giorgi, F. \& Lionello, P.; 2008. Climate change projections for the Mediterranean region. Global Planet Change 63: 90-104.

Harvell, C.D.; Mitchell, C.H.E.; WARD, J.R.; Altizer, S.; Dobson, A.P.; OstFeld, R.S. \& SAMUEL, M.D.; 2002. Climate warming and disease risks for terrestrial and marine biota. Science 296: 2158-2162. 
La Porta, N.; Capretti, P.; Thomsen, I.M.; Kasanen, R.; Hietala, A.M., \& Von WeisSENBERG, K.; 2008. Forest pathogens with higher damage potential due to climate change in Europe. Can. J. Plant Pathol. 30: 177-195.

Manion, P.D.; 1991 Tree Disease Concepts. Prentice Hall. Englewood Cliffs, $\mathrm{Nj}$.

Meehl, G.A.; Stocker, T.F.; Collins, W.D.; Friedlingstein, P.; Gaye, A.T.; Gregory, J.M.; Kitoh, A.; KnUTTI, R.; MurPhy, J.M.; NODA, A.; RAPER, S.C.B.; WATTERSON, I.G.; WeAVER, A.J. \& ZHAO, Z.-C.; 2007. Global Climate Projections. In: Climate Change 2007: The Physical Science Basis. In: Solomon, S., D. Qin, M. Manning, Z. Chen, M. Marquis, K.B. Averyt, M. Tignor \& H.L. Miller (eds.), Contribution of Working Group I to the Fourth Assessment Report of the Intergovernmental Panel on Climate Change. Cambridge University Press. Cambridge, United Kingdom and New York.

Ministerio DE Agricultura, Pesca Y Alimentación; 2005. 1154 Real Decreto. $58 / 2005$, de 21 de enero, por el que se adoptan medidas de protección contra la introducción y difusión en el territorio nacional y de la Comunidad Europea de organismos nocivos para los vegetales o productos vegetales, así como para la exportación y tránsito hacia países terceros. BOE 22 de enero de 2005: 2583-2565.

Page, W.G.; Jenkins, M.J. \& AleXander, M.E.; 2013. Foliar moisture content variations in lodgepole pine over the diurnal cycle during the red stage of mountain pine beetle attack. Environ. Modell. Softw. 49: 98-102.

REINFFORCE. INSTITUTE EUROPEEN DE LA FôRet Cultive (IEFC); 2013. Le réseau pour la gestion durable des forêts cultivées.
Project Interreg IV RÉseau INFrastructure de recherche pour le suivi et l'adaptation des FORêts au Changement climatiquE (REINFFORCE). http://reinfforce.iefc.net/

ReQue-KIlChenmann, J.A.; 2013. Patente de Invención número ES 2383420 B1. Protector de semillas para siembra en trabajos de forestación. http://bopiweb.com/elemento/829172/. Fecha de concesión: 16-04-2013.

Santini, A.; Ghelardini, L.; De Pace, C.; DesPRez-Loustau M.L.; Capretti, P.; ChandeLIER, A.; Cech, T.; Chira, D.; Diamandis, S.; GaitnieKs, T.; Hantula, J.; HoldenRIEDER, O.; JANKOVSKY, L.; JUNG, T.; JURC, D.; KIRISITS, T.; KunCA, A.; LygIS, V.; MALECKA, M.; Marcais, B.; SChMitz. S.; SChumacher, J.; Solheim, H.; Solla, A.; Szabò, I.; Tsopelas, P.; VAnNini, A.; VetTraino, A.M.; WoODWARD, S.; WebBer, J. \& StenLID, J.; 2013. Biogeographic patterns and determinants of invasion by alien forest pathogenic fungi in Europe. New Phythologist 197: 238-250.

Sturrock, R.N.; Frankelb, S.J.; Brown, A.V.; Hennond, P.E.; Kliejunasb, J.T.; Lewise, K.J.; Worrall, J.J. \& WoOD, A.J.; 2011. Review: Climate change and forest diseases. Plant Pathol. 60: 133-149.

The Montesclaros Declaration; 2011. IUFRO The Global Network for Forest Science and Cooperation. Division 7 - Forest Health. The Montesclaros Declaration. http://www.iufro.org/science/divisions/division-7/70000/publications/montesclarosdeclaration/

YAÑEZ-LÓPEZ, R.; TORRES-PACHECHO, I.; Guevara-GonZÁlez, R.G..; HERnÁN-DEZZUL, M.I.; QUIJANO-CARRANZA, J.A. \& RicOGARCÍA, E.; 2012. The effect of climate change on plant diseases. Afr. J. Biotechnol. 11(10): 2417-2428. 